\title{
Julgamentos de Plausibilidade e Reações Emocionais a Desculpas ${ }^{1}$
}

\author{
Renan Benigno Saraiva \& Fabio Iglesias*
}

Universidade de Brasília, Brasília, Brasil

\begin{abstract}
RESUMO
Desculpas são frequentemente utilizadas como forma de minimizar atribuições de causalidade interna após falhas na interação social. O objetivo deste trabalho foi investigar julgamentos de plausibilidade e reações emocionais a desculpas, assim como sua aceitabilidade. Participaram da pesquisa 155 estudantes universitários, que responderam a um instrumento contendo cenários com pedidos de desculpas para diversas situações de seu cotidiano. Foram julgadas mais plausíveis e geraram reações emocionais mais positivas as desculpas que envolveram argumentos percebidos como legítimos, seguidas de situações vistas como controláveis e por último as de causa interna. Os resultados são discutidos frente à literatura de gerenciamento de impressão e de sua eficácia para reestabelecer a harmonia em relações sociais informais.
\end{abstract}

Palavras-chave: desculpas; gerenciamento da impressão; atribuição de causalidade.

\begin{abstract}
Judgments of Plausibility and Emotional Reactions to Excuses

Excuses are frequently used to minimize internal causal attributions when social obligations are not fullfilled. This research investigated judgments of plausibility and emotional reactions to excuses, as well as their acceptability. One hundred and fifty five college students gave their ratings on several scenarios of excuse giving for routine situations. Situations involving motives perceived as legitimate received higher scores of plausibility and positive emotional reactions, followed by situations perceived as controllable, and those of internal causes. Results are discussed in the light of the impression management literature and its efficacy in maintaining informal bondings.
\end{abstract}

Keywords: excuses; impression management; causal attribution.

A boa navegação no mundo social depende de que se possa desenvolver uma autoimagem positiva. Para mantê-la, é frequentemente necessário que as pessoas afastem de si mesmas a culpa sobre suas ações inapropriadas, atribuindo-as a fatores externos e que possam parecer estar fora de seu controle. A mesma lógica se estende aos processos de gerenciamento de impressão investigados em psicologia social (Schlenker, 2012), sendo que em muitos idiomas o significado de palavras como "desculpa" (des-culpa) é antes de tudo um pedido para que o destinatário alivie o sentimento de culpa daquele que o emite.

Desculpas envolvem uma tentativa consciente de comunicar a outras pessoas uma redução da responsabilidade pessoal e tentar minimizar a raiva ou punição que se poderia receber delas (Weiner, 1995, 2006).
Nem toda tentativa de pedir desculpas é suficiente, entretanto, para reestabelecer as relações interpessoais afetadas ou para justificar as falhas cometidas. Essas estratégias podem ser usadas de maneira trivial e percebidas como falsas, mal intencionadas ou hipócritas. Assim, é fundamental investigar os fatores que influenciam o julgamento de um pedido de desculpas por quem o recebe, definindo sua aceitabilidade e considerando as potenciais aplicações desse conhecimento (Kruglanski \& Sleeth-Keppler, 2007). Da mesma forma, é importante conhecer os tipos de desculpas que podem efetivamente cumprir sua finalidade e aqueles que podem gerar reações ainda mais negativas. $\mathrm{O}$ objetivo deste trabalho foi analisar como as pessoas reagem a diferentes pedidos de desculpa em diversas situações, para investigar seus julgamentos e suas motivações.

\footnotetext{
Endereço para correspondência: Fabio Iglesias - iglesias@unb.br
} 
O gerenciamento de impressão, também conhecido como autoapresentação, pode ser definido como a forma pela qual os indivíduos expõem suas atividades a outras pessoas no cotidiano (Goffman, 1959/2002). Também pode ser mais espeficamente definido como os meios que o sujeito utiliza para regular a impressão que outros formam a seu respeito, com as funções de influenciá-los e reforçar a identidade pessoal (Leary, 1996), sendo especialmente utilizados pelos que desejam manter uma alta autoestima (Baumeister, Tice, \& Hutton, 1989). Entre os processos investigados nessa temática, incluem-se o uso de súplica, engraçamento, intimidação e autodesvantagem (Myers, 2000). É um fenômeno com ampla tradição de pesquisa na literatura internacional, mas que ainda encontra poucos trabalhos sistemáticos no Brasil (Araújo \& Pilati, 2009).

As pessoas têm um interesse contínuo em saber como são percebidas e avaliadas, deste modo comumente gerenciam seu comportamento em função de preservar uma impressão pessoal positiva tanto para outros quanto para si mesmas (Schlenker, 1980). Para Snyder, Higgins e Stucky (1983), as desculpas funcionam como um mecanismo desse gerenciamento de impressão, servindo como estratégia para proteger as pessoas de suas falhas, afastando de si a responsabilidade pelos seus comportamentos desfavoráveis ou socialmente indesejáveis.

Também se deve reconhecer que o tipo de mecanismo associado ao pedido de desculpas, entre outras lógicas que afastam a responsabilidade das pessoas das suas falhas, pode ser algo benéfico e até saudável (Snyder \& Higgins, 1988). As desculpas preservam o autoconceito dos indivíduos e aliviam efeitos negativos relacionados a seus resultados culposos (Taylor \& Brown, 1988). Pedir desculpas também protege a autoestima, livrando o indivíduo de possíveis julgamentos que contradigam sua autoimagem (Baumeister, 1993; Higgins \& Pittman, 2008). Na perspectiva do cometimento de transgressões que envolvem vítimas diretas e que tendem a produzir maior culpa em seus agentes, Bandura, Barbaranelli, Caprara e Pastorelli (1996) especificaram oito processos psicológicos pelos quais as pessoas tentam se desengajar situacionalmente de seus próprios padrões morais. Chamados de mecanismos de desengajamento moral, descrevem a utilização de recursos de justificação moral, eufemismo, comparação vantajosa, difusão da responsabilidade, deslocamento da responsabilidade, distorção das consequências, desumanização e atribuição da culpa. Aplicados ao contexto dos pedidos de desculpas, podem-se conceber facilmente maneiras de como mini- mizar a sentimento de responsabilidade pessoal pelo ocorrido, de como procurar diminuir sua importância, compará-lo com falhas maiores ou mesmo atribuir a responsabilidade à própria vítima (Iglesias, 2008).

\section{Atribuição de causalidade e desculpas}

A teoria da atribuição de causalidade, por meio de seus variados modelos, é a abordagem que substancia a maior parte dos trabalhos sobre desculpas em psicologia social (Basgall \& Snyder 1988; Snyder \& Higgins, 1988; Weiner, Folkes, Amirkhan, \& Verette, 1987). As explicações que os indivíduos dão, tanto para os seus próprios comportamentos quanto para os de outras pessoas, constituem o objeto de análise destas teorias (Heider, 1944, 1958), focando o tipo de atribuição como uma variável dependente. Nota-se, entretanto, que os trabalhos que geram maior valor heurístico são amparados em modelos que tratam a atribuição de causalidade como uma variável independente, que determina, por sua vez, certos tipos de reações, como no caso dos pedidos de desculpas.

Weiner (1995) usou a metáfora "o mundo é um tribunal" para expor sua teoria da conduta social: o ser humano está constantemente julgando os outros e a si mesmo e, para tanto, procura determinar a responsabilidade por atos cometidos. Esse autor identificou elementos fundamentais para analisar as atribuições de causalidade como uma variável independente: a estabilidade em que um efeito varia ao longo do tempo (percebido como estável versus instável), o lócus de controle (externo versus interno) e a controlabilidade da causa (controlável versus incontrolável). Diversos estudos têm mostrado que conforme as atribuições de causalidade se diferem nessas dimensões, configuram-se também diferentes reações (positivas ou negativas) diante do comportamento analisado.

Há uma tendência evidente nas pesquisas sobre atribuição de causalidade, mostrando que quanto mais externa for a causa do comportamento, ou seja, quanto menos participação o indivíduo tiver no comportamento desfavorável, menor será a atribuição negativa a ele associada. Logo, quanto mais uma desculpa move a causa do comportamento a fatores externos, mais eficiente ela pode ser (Snyder \& Higgins, 1988). Weiner et al. (1987) deram ênfase ao aspecto emocional dos pedidos de desculpas, destacando a reação emocional das pessoas que as recebem. Desculpas que sinalizam lócus interno, controlabilidade e intencionalidade na falha ("Não fui à reunião porque não quis") tendem a provocar sentimentos de raiva, enquanto são mais facilmente perdoáveis as desculpas de lócus externo, 
incontroláveis e não-intencionais ("Não fui à reunião porque meu carro quebrou no caminho").

São escassos no Brasil os estudos que usaram o modelo de Weiner com a perspectiva de tratar as atribuições como variáveis antecedentes no delineamento da pesquisa. Pilati (2011) investigou o efeito da proximidade social e de normas sociais no comportamento prosocial e verificou que a incontrolabilidade percebida pelos respondentes gerou maior empatia e maiores níveis de ajuda. Rodrigues e Assmar (2003) avaliaram a relação de tipos de atribuição e bases de poder, mostrando que punições mais leves são aplicadas a situações vistas como menos controláveis. Algumas outras pesquisas, embora publicadas no Brasil e usando o mesmo modelo, utilizaram amostras de participantes portugueses (Sousa, Rosado, \& Cabrita, 2008) ou foram centradas especificamente em temáticas escolares, como, por exemplo, as atribuições de alunos ao seu fracasso (Ferreira et al., 2002). Trabalhos empíricos que tratam especificamente dos pedidos de desculpas são poucos até mesmo na literatura internacional, ainda mais quando o foco de análise é o efeito negativo de se pedir desculpas (Tyler \& Feldman, 2007).

Considerando os elementos teóricos, metodológicos e aplicados que foram revisados, este estudo foi planejado para testar a aceitabilidade de diferentes tipos de desculpas, em função das atribuições de causalidade que o respondente faz. Foi hipotetizado que situações em que um indivíduo falhou por motivos internos e controláveis são classificadas como desculpas pouco plausíveis e geram sentimentos de raiva. Por outro lado, hipotetizou-se que desculpas de motivos externos e incontroláveis serão classificadas como desculpas mais plausíveis, gerando sentimentos de empatia.

Grande parte dos motivos por trás da escassez de estudos nessa temática deve-se a fatores metodológicos. Problemas de ordem ética, associados a restrições no uso de procedimentos de engano em experimentos ou no uso de ocorrências naturais acabam limitando a condução de pesquisas envolvendo transgressões sociais em situações mais realistas. Isso faz com que os estudos tenham uma natureza mais correlacional (Kimmel, 2004; Wilson, Aronson, \& Carlsmith, 2010), baseados em medidas de autorrelato. Como o problema de se trabalhar com situações reais em campo é de difícil resolução, preferiu-se o uso de cenários experimentais que simulam essas situações, como uma alternativa mais viável, tipicamente utilizada na literatura da área.

\section{MÉTODO}

\section{Participantes}

Participaram do estudo 155 estudantes universitários (78 mulheres), com média de 20,79 anos de idade $(\mathrm{DP}=4,94)$. Outras variáveis sociodemográficas, tais como religião ou renda, não foram utilizadas como critérios de seleção, considerando o que os estudos revisados têm especificado, assim como informações específicas sobre a área de ensino dos estudantes.

\section{Instrumentos}

A primeira etapa do estudo adotou uma perspectiva mais indutiva e qualitativa. Foi feito um levantamento a fim de apontar alguns pedidos de desculpas mais utilizados no cotidiano para que os dados da pesquisa se aproximassem mais do comportamento real. Para tanto, foram apresentadas quatro situações em um questionário para as quais os participantes deveriam formular pedidos de desculpas. Nestas situações foram narrados cenários fictícios de transgressão social, tal como: "Você está atrasado 45 minutos para um compromisso muito importante. Que justificativa você daria para o atraso?". Participaram desta etapa 10 estudantes universitários (seis mulheres), totalizando o elenco de 40 desculpas diferentes. Foram selecionadas a partir destas as que mais se repetiram (ou que foram bastante semelhantes) e que fossem mais aplicáveis ao cotidiano dos participantes.

Os dados do levantamento preliminar deram base à elaboração do instrumento. Seu objetivo era identificar quais as diferenças entre as desculpas em relação às seguintes dimensões: aceitabilidade (se a desculpa é aceitável ou não), plausibilidade (o quanto a desculpa é plausível), atribuições de responsabilidade (se quem falhou é ou não responsável pela falha cometida) e emoções (se o individuo sente raiva ou empatia diante do pedido de desculpas). Foram apresentados três tipos de situações em que um personagem fictício falhava com o respondente e para cada uma delas havia três diferentes pedidos de desculpas. Cada uma destas desculpas deveria ser analisada em escalas de 1 a 9, referentes à plausibilidade (não plausível - plausível), responsabilidade (responsável - não responsável) e emoções relacionadas (raiva - empatia), e ainda era perguntado se o participante iria aceitá-las em uma situação real (como variável dicotômica do tipo sim ou não). 


\section{Procedimentos}

Os participantes foram abordados individualmente no campus de uma universidade e convidados a participar da pesquisa, apresentada como um estudo sobre pedidos de desculpas e relações interpessoais. Após breve instrução, os participantes receberam caneta e uma prancheta para o preenchimento do instrumento. Atendendo-se a orientações de ordem ética, os respondentes consentiram previamente sua participação anônima e depois de terminado o preenchimento do questionário receberam uma breve explicação sobre a pesquisa e suas hipóteses gerais. O tempo médio de resposta ao questionário foi de cerca de cinco minutos para cada participante.

\section{RESULTADOS}

Considerando as categorias da escala de medida utilizada e o objetivo inicial de submeter os itens a testes paramétricos, o banco de dados foi submetido a um processo de análise exploratória para verificação de pressupostos, incluindo a eventual presença de casos faltosos, extremos ou outros problemas de distribuição. Como foram encontrados apenas alguns casos com resposta faltosa, adotou-se um procedimento de exclusão pair-wise quando apropriado. A análise de gráficos boxplot indicou a presença de casos extremos em algumas das variáveis de resposta, no entanto não foram considerados como tais, uma vez que a distância entre esses casos e o resto da distribuição não pareceu ameaçadora e as escalas contêm apenas nove categorias. Testes Komolgorov-Smirnov se revelaram significativos ao testar a normalidade de todas as variáveis escalares, mas como a análise fatorial é geralmente robusta à falta de normalidade (Hair, Anderson, Tatham, \& Black, 2005), esse procedimento foi mantido, em que pese também que pode ter sido reflexo do tamanho da amostra utilizada.
Os itens escalares foram todos submetidos a uma análise de componentes principais, que revelou indíces de fatorabilidade baixos e inadequados para se encontrar uma solução fatorial minimamente razoável. Verificou-se, entretanto, que os itens de percepção de responsabilidade é que estavam gerando problemas de correlação entre as variáveis, tendo-se optado por excluí-los dessa análise. Assim, o procedimento incluiu apenas os itens de plausibilidade e reações emocionais dos participantes aos pedidos de desculpa. Uma análise dos componentes principais $(\mathrm{KMO}=0,68) \mathrm{com}$ rotação oblíqua desses itens remanescentes sugeriu uma solução tridimensional com ponto de corte de 0,35. A classificação dos itens e suas cargas fatoriais são apresentadas na Tabela 1. O primeiro componente, nomeado como Desculpas Legítimas (com 6 itens e alfa $=0,79$ ), agrupou desculpas em que o indivíduo apresenta uma justificação que parece externa e incontrolável como motivo da sua falha (ex: "Desculpe-me, mas tenho uma prova muito importante e não poderei ir pois estarei estudando"). O segundo componente, Desculpas Controláveis (com 7 itens e alfa $=0,77$ ), agrupou desculpas que também possuem um elemento de externalidade. Diferente do primeiro componente, as desculpas neste segundo componente parecem mais passíveis de controle pelo indivíduo, já que ele poderia através de outros meios evitar os elementos externos que o levaram a cometer a falha (ex: "Desculpeme o atraso! O trânsito estava congestionado!"). Finalmente, o terceiro componente, nomeado como Desculpas Internas (com 5 itens e alfa $=0,67$ ), agrupou desculpas com características claras de internalidade, em que a culpa encontra-se predominantemente no indivíduo que cometeu a falha (ex: "Desculpe-me o atraso! Eu encontrei uma amiga no caminho, fiquei conversando com ela e por isso perdi a hora"). 
Tabela 1

Cargas fatoriais da análise dos componentes com rotação oblíqua para os julgamentos de plausibilidade e reação emocional aos tipos de desculpas

\begin{tabular}{|c|c|c|c|}
\hline Desculpas & Legítima & Controlável & Interna \\
\hline Mas tenho uma prova muito importante no dia seguinte e vou ter que ficar estudando (P) & ,767 & & \\
\hline Não tenho dinheiro porque fui demitido (E) & ,729 & & \\
\hline Mas já tenho um compromisso marcado pra esse dia com outro amigo (P) & ,721 & & \\
\hline Mas já tenho um compromisso marcado pra esse dia com outro amigo (E) & ,707 & & \\
\hline Não tenho dinheiro porque fui demitido $(\mathrm{P})$ & 630 & & \\
\hline Mas tenho uma prova muito importante no dia seguinte e vou ter que ficar estudando (P) & ,593 & & \\
\hline Encontrei uma amiga no caminho e fiquei conversando com ela, por isso perdi a hora (E) & & ,760 & \\
\hline Esqueci de pagar a fatura da internet e não deu para pesquisar a minha parte no trabalho $(\mathrm{E})$ & & ,744 & \\
\hline A semana foi corrida pra mim, tive muitas provas e fiquei sem tempo para fazer o trabalho (E) & & ,727 & \\
\hline Encontrei uma amiga no caminho e fiquei conversando com ela, por isso perdi a hora (P) & & ,697 & \\
\hline A semana foi corrida pra mim, tive muitas provas e fiquei sem tempo para fazer o trabalho $(\mathrm{P})$ & & ,560 & \\
\hline Esqueci de pagar a fatura da internet e não deu para pesquisar a minha parte no trabalho $(\mathrm{P})$ & & ,499 & \\
\hline O trânsito estava horrível e congestionado (E) & & ,395 & \\
\hline Fiquei de cama a semana toda. Realmente não tinha como pesquisar qualquer coisa (P) & & & ,824 \\
\hline Fiquei de cama a semana toda. Realmente não tinha como pesquisar qualquer coisa (E) & & & ,608 \\
\hline O trânsito estava horrível e congestionado $(\mathrm{P})$ & & &, 551 \\
\hline Tive que buscar meu irmão que tinha acabado de bater o carro antes de vir pra cá (E) & & & , 400 \\
\hline Tive que buscar meu irmão que tinha acabado de bater o carro antes de vir pra cá $(\mathrm{P})$ & & & ,484 \\
\hline
\end{tabular}

Nota. Foram omitidas as cargas menores que o ponto de corte de $0,35 . \mathrm{E}=$ Reação emocional; $\mathrm{P}=$ Plausibilidade.

Como os três componentes gerados apresentaram distribuição normal, eles foram analisados de forma paramétrica. Uma análise multivariada da variância para medidas repetidas mostrou que Desculpas Legítimas receberam as maiores médias de avaliação, seguidas de Desculpas Controláveis e de Desculpas Internas, $F(2,150)=221,65, p<0,001, \eta_{\mathrm{p}}^{2}=0,75$. Os resultados também mostraram uma interação significativa dessas variáveis com o sexo do respondente.
Embora homens e mulheres tenham atribuído um valor maior de plausibilidade e maior empatia às desculpas externas e incontroláveis (Desculpas Legítimas), as mulheres consideraram essas desculpas mais legítimas que os homens. Por outro lado, os homens consideraram as desculpas relacionadas a internalidade (Desculpas Internas) mais legítimas do que as mulheres, $F(2,150)=4,17, p=0,02, \eta_{\mathrm{p}}^{2}=0,05$. Esses dados são ilustrados na Figura 1.

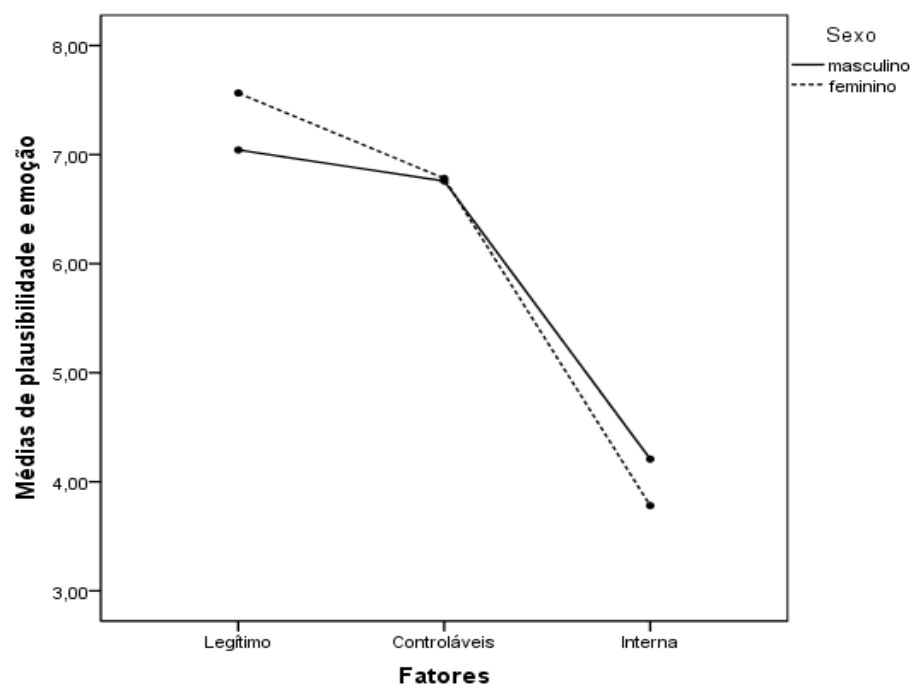

Figura 1. Julgamentos conjuntos de plausibilidade e reação emocional de cada sexo para as dimensões dos pedidos de desculpas. 
Não foram verificadas diferenças sistemáticas em função da idade, dada a caracterização mais homogênea da amostra de estudantes. Como uma medida adicional de reação às desculpas, verificou-se de forma dicotômica se os respondentes as aceitariam em cada uma das situações apresentadas nos cenários. Esses dados mostraram que dentre os nove tipos de desculpas analisadas, seis delas seriam aceitas por mais de 84\% dos participantes. Essas tendências dispensaram o uso de análises não-paramétricas para testar a associação entre as variáveis, dado o considerável desequilíbrio nas categorias de ocorrência. As desculpas com maior nível de internalidade (que espelham o componente Desculpas Internas) foram aceitas por menos de $50 \%$ dos participantes, independentemente do tipo de situação.

\section{DISCUSSÃO}

O objetivo desta pesquisa foi analisar as reações a diferentes tipos de pedidos de desculpas em situações cotidianas, tomando-se por base a teoria atribucional de Weiner (2006). Além dos julgamentos de plausibilidade e aceitabilidade dessas desculpas, foram consideradas também as reações emocionais previstas pelo modelo teórico. Os resultados mostraram que os tipos de desculpa avaliados como mais efetivos (Desculpas Legítimas) correspondem ao previsto no modelo de Weiner. Por serem desculpas em que o indivíduo expõe uma situação praticamente incontrolável como causa de sua falha, ele não é visto como agente principal e tende a ficar livre de possíveis punições. Já no segundo componente encontrado (Desculpas Controláveis) as desculpas sugerem que o motivo da falha não está propriamente no indivíduo, mas que ele poderia por outros meios ter evitado que a falha ocorresse, sendo julgadas como desculpas menos efetivas que as primeiras. Por fim, o terceiro componente (Desculpas Internas) revelou que desculpas em que o sujeito é visto como principal agente da falha não cumprem bem o papel de livrá-lo da culpa, recebendo reações emocionais negativas e possíveis punições. Essa associação entre desculpas e atribuições externas parece ser tão evidente que até mesmo crianças são capazes de identificá-la (Darby \& Schlenker, 1989).

Uma diferença encontrada entre os sexos merece discussão, ainda que tenha apresentado um tamanho de efeito pequeno. Os homens se mostraram menos tolerantes com as desculpas mais legítimas, enquanto as mulheres se mostraram menos tolerantes com as desculpas mais controláveis. Hirt, McCrea e Boris (2003) relataram o mesmo tipo de resultado, indican- do uma diferença entre os sexos na avaliação de comportamentos desfavoráveis. Estes autores encontraram, por exemplo, que mulheres se mostram menos tolerantes a desculpas falhas ou ilegítimas, independente do sexo de quem elas estão julgando. Trata-se de tendência sugestiva de avaliações que podem carregar diferentes significados morais, cujo exame deve conciliar tanto perspectivas do debate tradicional sobre emoção versus cognição, quanto do debate mais contemporâneo sobre processos automáticos versus explícitos em psicologia social (Haidt \& Kesebir, 2010).

Os dados referentes a atribuição de responsabilidade não foram significativos, o que pode ter sido um problema metodológico com o uso do termo "responsabilidade" no instrumento. Muitos participantes parecem ter interpretado o termo como um traço de personalidade daquele que está se desculpando, caracterizando-o como uma pessoa de caráter responsável ou não, ao invés de analisar se o indivíduo foi responsável somente pela falha cometida. Essas são limitações que o modelo de Weiner (2006) apresenta em suas adaptações culturais. O termo empatia, por exemplo, que expressa uma das reações emocionais do modelo, é frequentemente confundido por respondentes brasileiros como simpatia, embora tenham significados e focos distintos.

O estudo também possui algumas limitações referentes à fidelidade dos achados pela pesquisa frente ao comportamento real dos indivíduos. Restrições de ordem ética obviamente dificultam a condução de pesquisas de campo que envolvam situações de transgressão real ou outras temáticas semelhantes a deste estudo, tornando o autorrelato uma das poucas alternativas disponíveis para operacionalizar as variáveis. Uma vantagem relativa do estudo, entretanto, é que foram investigadas desculpas previamente listadas por respondentes (na primeira etapa de coleta) com o mesmo perfil dos respondentes dos cenários (na segunda etapade coleta). Isso conferiu inicialmente uma perspectiva mais indutiva à pesquisa, procurando compensar as críticas feitas a alguns autores que partem de situações que eles mesmos consideram relevantes e que não são necessariamente relevantes para os respondentes.

Ainda existe uma ampla agenda de pesquisa a ser desenvolvida sobre desaprovações e orientações punitivas após transgressões sociais e morais (Weiner, 2006). Os dados deste estudo contribuem para a interpretação do pedido de desculpas como um mecanismo de gerenciamento de impressão, utilizado para ameni- 
zar ou até mesmo anular tais punições. Quanto mais uma desculpa transferir a causa de um comportamento desagradável a fatores situacionais, melhor será sua função de livrar o indivíduo da culpa (Snyder \& Higgins, 1988). Evidencia-se que esse julgamento de responsabilidade não se deve somente a características de internalidade ou externalidade, como postuladas no trabalho original de Heider $(1944,1958)$ e posteriormente por autores como Kelley (1967), mas também a fatores de controlabilidade do indíviduo perante o evento ocorrido. Isso aumenta a importância, como identificado por Weiner et al. (1987) em seus estudos sobre causalidade e pedidos de desculpa, de se posicionar a atribuição de causalidade como uma variável independente nos delineamentos de pesquisa.

Darley \& Pittman (2003) argumentaram que a primeira reação de um indivíduo que causa algum tipo de dano a outro é buscar uma forma de compensar o dano causado, sendo tal reação impulsionada por um sentimento de remorso. Identificou-se nesta pesquisa que desculpas em que o indivíduo expõe uma situação fora de seu controle parecem amenizar o dano causado. Por outro lado, desculpas controláveis e inerentes ao infrator não parecem compensar o dano que este causou, podendo até mesmo piorar sua imagem social por meio de reações emocionais negativas. Entretanto, há de se considerar a possibilidade de que o uso constante de desculpas externas e incontroláveis pode parecer enganoso e ter efeitos negativos a longo prazo, sendo ainda uma questão empírica em aberto. Como as pesquisas sobre essa temática são praticamente inexistentes no Brasil, falta um conjunto de dados mínimos para que se possa averiguar influências culturais na interpretação e julgamento dos pedidos de desculpas. Isso parece ter sido feito apenas em estudos linguísticos, comparando-se, por exemplo, sua ocorrência em roteiros de filmes iberoamericanos (Monteiro, Lopes, \& Rebollo Couto, 2007). Apesar dessa pergunta empírica permanecer em aberto, o presente trabalho aponta que os princípios usados em tais julgamentos de desculpas no Brasil parecem ser os mesmos que nos países que possuem maior literatura acerca dessa temática. Deve-se atentar, no entanto, para o fato de que a cultura também pode influenciar os estilos de resposta em escalas Likert e similares, considerando-se o uso diferenciado de categorias extremas, intermediárias e a propensão a concordar ou discordar com itens (Hamamura, Heine, \& Paulhus, 2008). Estudos posteriores sobre essa temática podem explorar os efeitos negativos dos pedidos de desculpas, investigando o que ocorre nas avaliações da vítima quando tais pedi- dos são identificados como falsos ou desonestos. Outra possibilidade é investigar se o efeito negativo de desculpas internas e controláveis pode ser amenizado através de outros mecanismos de compensação de dano. Devem considerar também as diversas aplicações desse conhecimento, desde as esferas mais judiciais até as esferas mais cotidianas, como nas situações específicas que foram aqui investigadas.

\section{REFERÊNCIAS}

Araújo, M. E. B. O., \& Pilati, R. (2008). Gerenciamento de impressão nas entrevistas de seleção: Proposição de uma agenda de pesquisa. Psicologia: Organizações e Trabalho, 8(2), 121138.

Bandura, A., Barbaranelli, C., Caprara, G. V., \& Pastorelli, C. (1996). Mechanisms of moral disengagement in the exercise of moral agency. Journal of Personality and Social Psychology, 71, 364-374.

Basgall, J. A., \& Snyder, C. R. (1988). Excuses in waiting: External locus of control and reactions to success-failure feedback. Journal of Personality and Social Psychology, 54(4), 656-662.

Baumeister, R. F. (Ed.) (1993). Self-esteem: The puzzle of low self-regard. New York: Plenum.

Baumeister, R. F., Tice, D. M., \& Hutton, D. G. (1989). Selfpresentational motivations and personality differences in selfesteem. Journal of Personality, 57, 547-579.

Darby, B. W., \& Schlenker, B. R. (1989). Children's reactions to transgressions: Effects of the actor's apology, reputation, and remorse. British Journal of Social Psychology, 28, 353-364.

Darley, J. M., \& Pittman, T. S., (2003). The psychology of compensatory and retributive justice. Personality and Social Psycholy Review, 7(4), 324-336.

Ferreira, M. C., Assmar, E. M. L., Omar, A. G., Delgado, H. U., González, A. T., Silva, J. M. B., Souza, M. A., \& Cisne, M. C. F. (2002). Atribuição de causalidade ao sucesso e fracasso escolar: Um estudo transcultural Brasil-Argentina-México. Psicologia: Reflexão e Crítica, 15(3), 515-527.

Goffman, E. (2002). A representação do eu no cotiadiano. Petrópolis: Vozes. (Trabalho original publicado em 1959)

Haidt, J. \& Kesebir, S. (2010). Morality. In S. T. Fiske, D. T. Gilbert, \& G. Lindzey (Eds.), Handbook of social psychology (Vol. 2, pp. 797-832). Hoboken, NJ: Wiley.

Hair Jr., J. F., Anderson, R. E., Tatham, R. L., \& Black, W. C. (2005). Análise multivariada de dados. Porto Alegre: Bookman.

Hamamura, T., Heine, S. J., \& Paulhus, D. L. (2008). Cultural differences in response styles: The role of dialectical thinking. Personality and Individual Differences, 44, 932-942.

Heider, F. (1944). Social perception and phenomenal causality. Psychological Review, 51(6), 358-374. 
Heider, F. (1958). The psychology of interpersonal relations. New York: Wiley.

Higgins, E. T. \& Pittman. T. S. (2008). Motives of the human animal: Comprehending, managing, and sharing inner states. Annual Review of Psychology, 59, 361-385.

HIRT, E. R., MCCREA, S. M., \& BORIS, H. I. (2003). "I know you self-handicapped last exam": gender differences in reactions to self-handicapping. Journal of Personality and Social Psychology, 84(1), 177-193.

Iglesias, F. (2008). Desengajamento moral. In A. Bandura, R. G. Azzi \& S. Polydoro (Eds.), Teoria social cognitiva: Conceitos básicos (pp. 165-176). Porto Alegre: Artes Médicas.

Kelley, H. H. (1967). Attribution theory in social psychology. In D. L. Vine (Eds.), Nebraska symposium on motivation (pp. 192-238). Lincoln: University of Nebraska Press.

Kimmel, A. J. (2004). Ethical issues in social psychology research. In C. Sansone, C. C. Morf \& A. T. Panter (Eds.), Handbook of methods in social psychology (pp. 45-70). California: Sage.

Kruglanski, A. W., \& Sleeth-Keppler, D. (2007). The principles of social judgment. In A. W. Jruglanski \& E. T. Higgins (Eds.), Social psychology: Handbook of basic principles (pp. 116137). New York: Guilford.

Leary, M. R. (1996). Self-presentation: Impression management and interpersonal behavior. Boulder, CO: Westview.

Leary, M. R., Tchividjian, L. R., \& Kraxberger, B. E. (1994). Selfpresentation can be hazardous to your health: Impression management and health risk. Health Psychology, 13, 461-470.

Monteiro, F. A., Lopes, C. R. S., \& Rebollo Couto, L. (2007). Perdón, desculpa, desculpa aí: La expresión de las disculpas en el cine iberoamericano. Gragoatá, 22, 253-269.

Myers, D.G. (2000). Psicologia social. Rio de Janeiro: LTC.

Pilati, R. (2011). Cenários experimentais: Efeito sobre a emoção e o comportamento prosocial. Estudos de Psicologia (Natal), 16, $163-170$

Rodrigues, A. \& Assmar, E. M. L. (2003). Influência social, atribuição de causalidade e julgamentos de responsabilidade e justiça. Psicologia Reflexão e Crítica, 16(1), 191-201.

Schlenker, B. R. (1980). Impression management: The selfconcept, social identity, and interpersonal relations. Monterey, CA: Brooks/Cole.
Schlenker, B. R. (2012). Self-presentation. In M. Leary \& J. P. Tangney (Eds.), Handbook of self and identity (pp. 542-570). New York: Guilford Press.

Snyder, C. R. \& Higgins, R. L. (1988). Excuses: Their effective role in the negotiation of reality. Psychological Bulletin, 104, 23-35.

Snyder, C. R., Higgins, R. L., \& Stucky, R. J. (1983). Excuses: Masquerades in search of grace. New York: Wiley.

Sousa, P. M., Rosado, A. B., \& Cabrita, T. M., (2008). Análise das atribuições causais do sucesso e do insucesso na competição, em função do nível competitivo dos futebolistas portugueses. Psicologia: Reflexão e Crítica, 21, 203-211.

Taylor, S. E. \& Brown, J. D. (1988). Illusion and well-being: A social psychological perspective on mental health. Psychological Bulletin, 103, 193-210.

Tyler, J. M. \& Feldman, R. S. (2007). The double-edged sword of excuses: When do they help, when do they hurt? Journal of Social and Clinical Psychology, 26, 659-688.

Weiner, B. (1986). An attributional theory of motivations and emotion. New York: Springer-Verlag.

Weiner, B. (1995). Judgments of responsibility: A foundation for a theory of social conduct. New York: Guilford.

Weiner, B. (2006). Social motivation, justice, and the moral emotions: An attributional approach. Mahwah, NJ: Lawrence Erlbaum Associates.

Weiner, B., Folkes, V., Amirkhan, J., \& Verette, J. (1987). An attributional analysis of excuse giving: Studies of a naïve theory of emotion. Journal of Personality and Social Psychology, $52,316-324$

Wilson, T. D., Aronson, E., \& Carlsmith, K. (2010). The art of laboratory experimentation. In S. T. Fiske, D. T. Gilbert, \& G. Lindzey (Eds.), Handbook of social psychology (Vol. 1, pp. 5181). Hoboken, NJ: Wiley.

\section{Nota:}

1 Os autores agradecem o financiamento recebido do CNPq e da FINATEC, assim como a Carolina Bauchspiess, Carolina Cidade, Izabella Melo e Lorena Andreoli pelo papel no desenvolvimento do instrumento e da coleta de dados. 